\title{
Dark breather using symmetric Morse, solvent and external potentials for DNA breathing
}

\author{
Hernán Oscar Cortez Gutierrez ${ }^{1+}$, Milton Milciades Cortez Gutierrez ${ }^{2}$ i⿺ , Girady Iara Cortez Fuentes Rivera ${ }^{3}$, Liv Jois \\ Cortez Fuentes Rivera ${ }^{4}$, Deolinda Fuentes Rivera Vallejo ${ }^{3}$ \\ 1 Universidad Nacional del Callao, Facultad de Ciencias de la Salud, 306 Juan Pablo II Av, Callao, Bellavista, 07001, Peru \\ 2 Universidad Nacional de Trujillo, 555 Jr. Salaverry Urb. Mansiche, Peru \\ 3 Universidad Inca Garcilaso de la Vega, 890 San Felipe Av., Jesús María, Lima, Peru \\ 4 Universidad Privada San Juan Bautista, José Antonio Lavalle St., Distrito de Chorrillos, 15067, Peru
}

+ Corresponding author: Hernan Oscar Cortez Gutierrez, phone: +51 964353795, e-mail address: hcortez@unac.pe

\section{ARTICLE INFO}

Article history:

Received: April 29, 2018

Accepted: October 13, 2018

Published: December 5, 2018

\section{Keywords:}

1. Mobile dark breather

2. Center of energy

3. quantum thermodynamics

4. DNA breathing

ABSTRACT: We analyze the dynamics and the quantum thermodynamics of DNA in Symmetric-Peyrard-Bishop-Dauxois model (S-PBD) with solvent and external potentials and describe the transient conformational fluctuations using dark breather and the ground state wave function of the associate Schrodinger differential equation. We used the S-PBD, the Floquet theory, quantum thermodynamic and finite difference methods. We show that for lower coupling dark breather is present. We estimate the fluctuations or breathing of DNA. For the S-PBD model we have the stability of dark breather for $\mathrm{k}<0.004$ and mobile breathers with coupling $\mathrm{k}=0.004$. The fluctuations of the dark breather in the S-PBD model is approximately zero with the quantum thermodynamics. The viscous and external potential effect is direct proportional to hydrogen bond stretching.

\section{Introduction}

The deoxyribonucleic acid DNA is a thread-like chain of nucleotides carrying the genetic information of all organisms. The coding sequences for genes and regulatory information are located in DNA and is marginally stable and undergoes a "melting phase transition". There are many experimental ways to study the fluctuations or breathing of DNA: hydrogen exchange, formaldehyde probing, protein-nucleic acid interactions, DNA replication, DNA base analogue spectroscopy, single molecule DNA-protein interactions, two-dimensional fluorescence spectroscopy ${ }^{1}$. The interaction between the viscous potential and external forces prevent DNA to unzip perfectly but allows DNA to split at a certain distance from its original position ${ }^{2}$. S. Flach gives the theory of the "discrete breathers" and applications ${ }^{3}$.

R. S. Mackay investigates the Peyrard Bishop model for the study of nonlinear excitations travelling along the DNA chains ${ }^{4}$. J. Cuevas has results about breathing of DNA using the spatially localized oscillations or 'discrete breathers'. The mobility and breathing of DNA depends on the harmonic bifurcation ${ }^{6}$. The strong dependence on sequence, temperature and salt concentration for the breathing dynamics of DNA found here points 
at a good potential for applications and the effect of the viscous and external forces ${ }^{2,7}$.

First, the PBD model is introduced. It is then followed by the dynamical and the thermodynamic formulations. We show that mobile breather can lead to the observed breathing, but the amplitude of the breather is determinant for the transient conformational fluctuations of DNA. The results obtained in our simulation verify the existence of dark breather with the conditions describes by R.S. Mackay. The symmetric potential does not give a solution for the transition of the DNA. For that reason, it is necessary to investigate the effect of the solvent and external potentials. The calculation of hydrogen bond stretching using transfer integral operator and difference finite methods are presented.

\section{Materials and methods}

\subsection{Symmetric Morse Potential in the PBD model}

The biomechanics of DNA is represented by two degree of freedom $X_{n}$ and $Y_{n}$ which correspond to the displacement of the base pair from their equilibrium position along the direction of the hydrogen bonds connecting the two-base pair of nucleotides.

The studies of the Symmetric Peyrard-BishopDauxois (S-PBD) models that included the modified Morse potential was done by adding the absolute value:

$$
V(u)=\frac{1}{2}[\exp (-|u|)-1]^{2}
$$

where V = symmetric Morse Potential.

The profile of symmetric Morse potential can be seen in Figure 1.

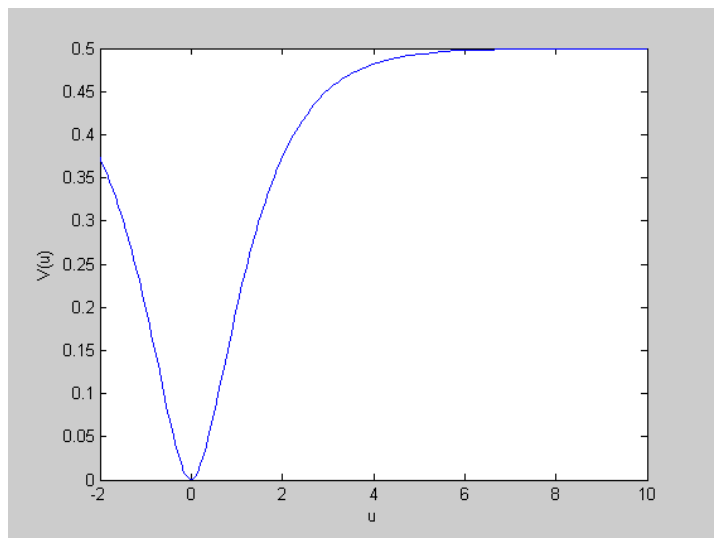

Figure 1. The symmetric Morse potential.

$$
H=\sum_{n=1}^{N} \frac{m}{2} \dot{u}_{n}^{2}+\sum_{n=1}^{N} \frac{K}{2}\left(u_{n}-u_{n-1}\right)^{2}+\sum_{n=1}^{N} V_{n}\left(u_{n}\right)
$$

where $\mathrm{N}=$ number of the pairs of bases; $\mathrm{K}=$ velocity $=u$ coupling constant; and $\mathrm{u}_{\mathrm{n}}=$ stretching of the hydrogen bonds $=\left(X_{n}-Y_{n}\right) / \sqrt{2}$.

\subsection{Dynamics of $S-P B D$}

The associated equations for Equation 4 are the system equations $(\mathrm{n}=1,2 \ldots \mathrm{N})$ :

$\ddot{u}_{n}+\operatorname{sign}\left(u_{n}\right)\left[e^{-2 / u_{n} I}-e^{-l u_{n} \prime}\right]+K\left(2 u_{n}-u_{n-1}-u_{n+1}\right)=0$

Using the approximation for the oscillator $\mathrm{n}$ and $\mathrm{T}=2 \pi / \mathrm{w}_{\mathrm{b}}$

$u_{n}=z_{n}^{0}+\sum_{k=1}^{k_{m}} 2 z_{n}^{k} \cos \left(k \omega_{b} t\right)$

and substituting in Eq. 3 one has:

$k^{2} \omega_{b}^{2} z^{2}{ }_{n}+V_{n}{ }^{k}+K\left(2 z^{k}{ }_{n}-z^{k}{ }_{n-1}-z^{k}{ }_{n+1}\right)=0$

which depends on the parameter $\mathrm{K}$, and $V_{n}^{\prime}{ }^{k}$ is the $k^{\text {th }}$ Fourier coefficient for the periodic Function $V^{\prime}\left(u_{n}(t)\right)$.

\section{Remark 1. One site dark breather}

The dark breather solution is obtained in conditions $(\mathrm{t}=0)$ where all the oscillators are at rest, but equally shifted from their equilibrium position, while the central one is at the rest. The codification for one site dark breather is $1,1, \ldots 1,0,1, \ldots, 1,1$.

In Fig. 2 the dark breather is depicted. This figure shows the numerical solution of equation (5). The second derivative of the symmetric Morse potential is given by:

$$
V^{\prime \prime}=\left\lfloor 2 e^{-2 / u_{n} /}-e^{-/ u_{n} /}\right\rfloor .
$$




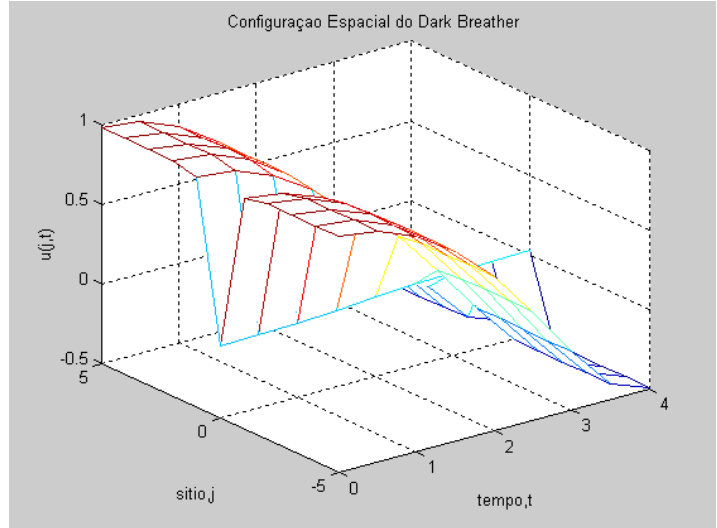

Figure 2. Spatial dark breather configuration of the symmetric Morse potential.

The dynamics of the DNA is a set of coupled oscillators, and the vibrational motion is equivalent to equation (3) which depends of the Symmetric Morse potential and constant $K$ of coupling.

The amplitude of the breather is determinant for the transient conformational fluctuations of DNA. In our case the Figure 2 give a small amplitude.

\subsubsection{Existence of Harmonic bifurcation}

We analyze the stability of the breather solution. Let us introduce a function $\tilde{u}_{\mathrm{n}}(\mathrm{t})=\mathrm{u}_{\mathrm{n}}(\mathrm{t})+\varepsilon_{\mathrm{n}}(\mathrm{t})$, where $u_{n}(t)$ is the periodic breather solution shown in Figure 2. The term $\varepsilon_{\mathrm{n}}(\mathrm{t})$ is a perturbation: $\tilde{\mathrm{u}}_{\mathrm{n}}(\mathrm{t})$ must satisfy the system (3) and expanding around $\mathrm{u}_{\mathrm{n}}(\mathrm{t})$ to first order (linearization), we obtain the following system of equations for $\varepsilon_{\mathrm{n}}(\mathrm{t})$

$$
\ddot{\varepsilon}_{n}+\left(V^{\prime \prime}\left(u_{n}(t)\right)\right) \varepsilon_{n}+K\left(2 \varepsilon_{n}-\varepsilon_{n-1}-\varepsilon_{n+1}\right)=0
$$

We can associate a monodrama my matrix for this equation with Fouquet multipliers ${ }^{5}$.

The solution is stable if the modules of Fouquet multipliers are one. The especial instability ("harmonic bifurcation") in our case happens when a pair of Fouquet multipliers merges at $\lambda=1$ and splits off circle onto the positive real axis in Fig. 3.

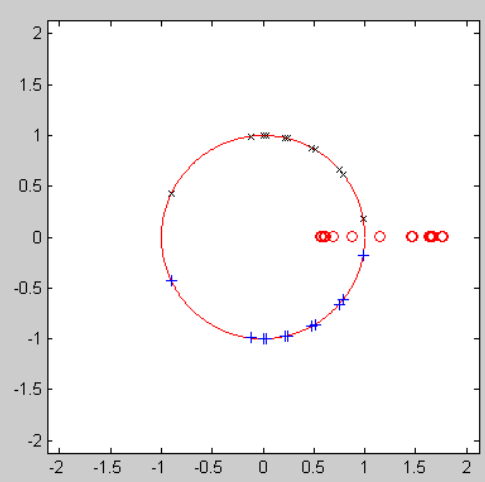

Figure 3. The instability "harmonic bifurcation" with the evolution of the Fouquet multipliers. Case SPBD model with the parameters: $K=0.004, \mathrm{w}_{\mathrm{b}}=0.8$ for the dark breather.

\subsubsection{Existence of Mobile breather}

For the coupling $K=0.004$ and $w_{b}=0.8$ there is a harmonic bifurcation. In this case we can construct a dark breather mobile. Once the system of equations (3) is worked out by Runge Kutta method for the Cauchy problem with the equations (3). We can use the Figure 2 for the initial conditions of the position and average speed of each position " $n$ " respect to the harmonic oscillation corresponding to the DNA.

The center of energy of the breather mobile is given by ${ }^{5}$

$$
X_{E}=\sum_{n=1}^{N} n H_{n}^{d} / H
$$

where the density energy has the form

$$
H_{n}^{d}=\frac{1}{2} \dot{u}_{n}^{2}+\frac{K}{4}\left(u_{n}-u_{n-1}\right)^{2}+\frac{K}{4}\left(u_{n+1}-u_{n}\right)^{2}+V_{n}\left(\sqrt{2} u_{n}\right)
$$

It is very important the initial velocity of the $\mathrm{BM}$ for the displacement a long of sites of DNA and can be produced of DNA breathing.

This parameter initial velocity $v(0)$ is transcendental for DNA breathing.

Remark 2. Initial velocity and the perturbation velocity

We can use the profiles of the stationary dark breather obtained from equations (3). The velocity is a vector which the components are given by

$v_{n}(0)=\left(u_{n+1}(0)-u_{n-1}(0)\right) / 2$ 
We can define a perturbation velocity in terms of the parameter $\lambda$. The components of this vector perturbation $V$ are given by

$$
V_{n}(0)=\lambda\left(v_{n}(0) / v\right)
$$

Where $v$ is the norm of the vector of the components $v_{n}(0)$.

Remark 3. The Cauchy problem is given by

$$
\ddot{u}_{n}+V^{\prime}\left(u_{n}\right)+K\left(2 u_{n}-u_{n-1}-u_{n+1}\right)=0
$$

Initial conditions: $u(0)=$ profiles of the solutions of the Figure 2. The velocities are given by the expression (10) with $\lambda=0.1$. We can obtain the solutions of the equations using initial condition with the software Fortran (for a review, see ref. 9).

\subsection{Quantum Thermodynamics of S-PBD}

The evaluation of the partition of equation (2) using the transfer integral operator method in the thermodynamic limit reduces to solving the pseudo-Schrodinger equation (12):

$$
\begin{aligned}
& \left\{-1 /\left(2 \beta^{2} K\right) d^{2} / d u_{n}^{2}+U\left(u_{n}, \beta\right)\right\} \psi\left(u_{n}\right)=\varepsilon \psi\left(u_{n}\right) \\
& U\left(u_{n}, \beta\right)=V\left(\sqrt{2} u_{n}\right)+(1 / 2 \beta) \ln (\beta K / 2 \pi) \\
& \beta=1 /\left(K_{B} T\right)
\end{aligned}
$$

We use the symmetric Morse potential. The fluctuations or breathing of DNA can be performed numerically using the finite difference methods. Firstly, we obtain the ground state wave function of equation (12). For estimate the mean value of the fluctuations we use the formula:

$$
<u>=\int_{-\infty}^{+\infty} \psi^{2}{ }_{0} u d u
$$

The ground state wave function for the symmetric Morse potential is symmetric in consequence the mean value of the fluctuations is approximately zero (for a review, see ref. 12).

In Figure 4 is depicted the example of the ground state wave function for the symmetric Morse potential.

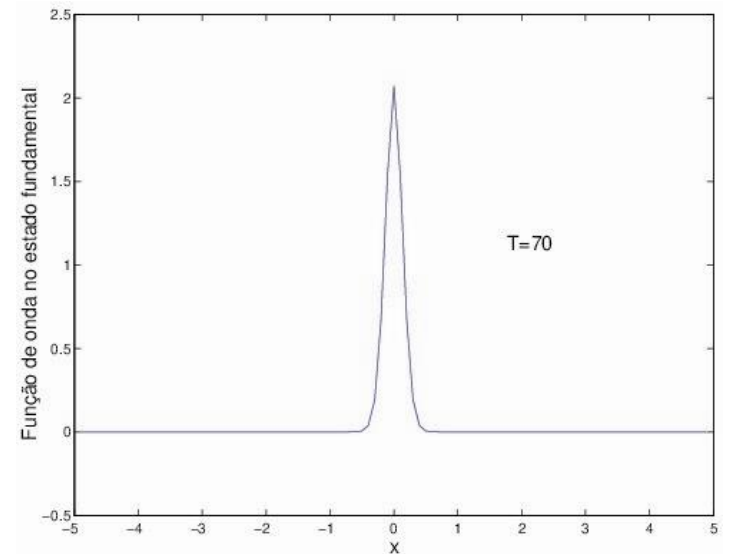

Figure 4. Ground state wave function for the symmetric Morse potential with the control parameter Temperature $=70^{\circ} \mathrm{K}$.

\subsubsection{Quantum Thermodynamics of S-PBD with solvent and external potentials}

We can consider the new potential for the equation (13):

$$
\begin{gathered}
U\left(u_{n}\right)=V\left(\sqrt{2} u_{n}\right)+V \text { solvent }\left(u_{n}\right)+V_{0} \\
\exp \left(-0.1 u_{n}^{2}\right)+(1 / 2 \beta) \ln (\beta K / 2 \pi)
\end{gathered}
$$

The solvent potential is given by: $\mathrm{V}$ solvent $=$ $0.04 * v * \tanh \left(\mathrm{u}_{\mathrm{n}} / 5-1\right)$. In Figure 5, it is depicted the example of the solvent potential.

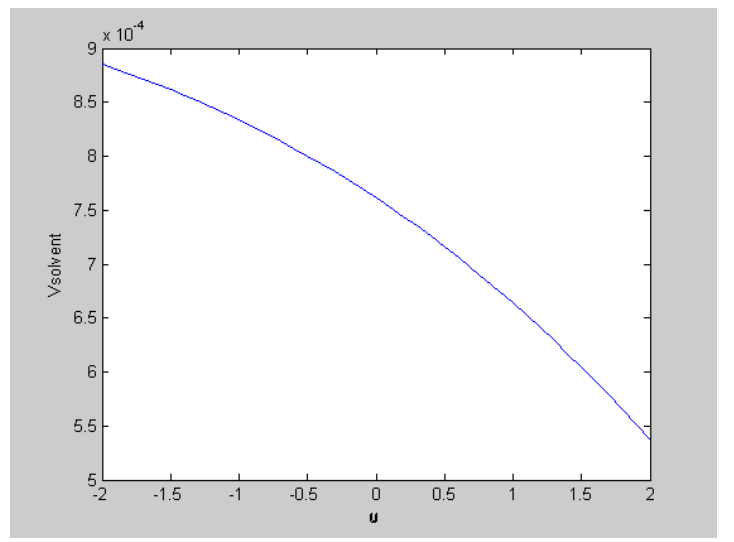

Figure 5. The solvent potential with the control parameter $\mathrm{v}=0.025$.

For the symmetric Morse potential in the SPBD Model we can get many values of the melting temperatures. For example, for $\mathrm{T}=270 \mathrm{~K}$ and the control parameter $v=0.001, V_{0}=0.005$ the mean value of the fluctuations $\langle u\rangle=1.9586 \AA$. The hydrogen bond stretching as a function of 
temperature gives a melting temperature depicted in Figure 6.

Bustamante $^{13}$ has the interplay between the "DNA breathing" with the viscosity coefficient of the medium.

a)

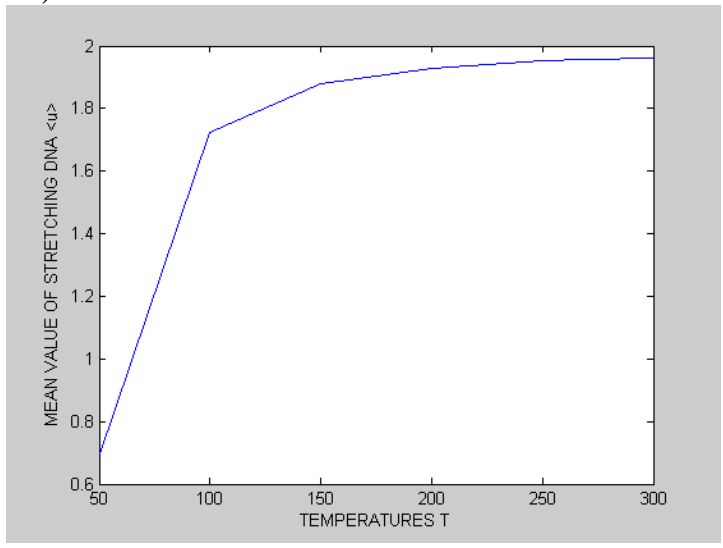

b)

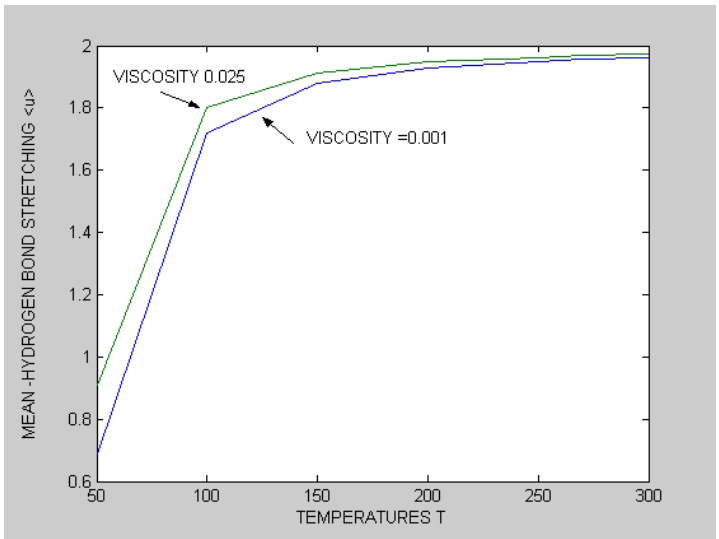

Figure 6. The hydrogen bond stretching as a function of temperature for external.

Potential $V=V_{0} \exp \left(-0.1 u^{2}\right), V_{0}=0.005$ and solvent potential with the viscosity control parameter $v: a$ ) $v=0.001$ and $b$ ) $v=0.025$.

\section{Results and discussion}

The solutions of the dynamical equations (5) give the dark breather mobile. We have the mobile breather using the center of energy for the initial velocity of 0.1 . This method is based on the literature $^{6,8}$.

We have obtained harmonic bifurcation using the symmetric Morse potential with the parameter $K=0.004$.
We have obtained the Eigen functions of the pseudo-Schrodinger equation (12) to demonstrate that the mean value breathing of DNA is zero. The analysis is based on the reference ${ }^{12}$.

For the symmetric Morse potential in the SPBD Model, we can get the melting temperature for $\mathrm{T}=270 \mathrm{~K}$, control viscosity parameter $v=$ 0.001 and the constant of the external potential $V_{0}$ $=0.005$. For these values the mean value of the fluctuations $\langle u\rangle$ is $1.9586 \AA$. In this case, we can get the DNA breathing with the variations of temperatures (Figure 6a).

Figure $6 \mathrm{~b}$ indicates that mean value of stretching $\langle u\rangle$ is direct proportional to the coefficient of viscosity. The increase of the viscosity will increase the hydrogen bond stretching. The viscous and external potential effect is direct proportional to hydrogen bond stretching. For $V_{0}=0.5$ the mean value of hydrogen bond is $\langle u\rangle=3.82$ with the temperature $\mathrm{T}=270 \mathrm{~K}$ and viscosity $v=0.025$.

The Figure 6 shows that for $\mathrm{T}>150 \mathrm{~K}$ the viscous force is not important for the DNA breathing. This result is similar to that obtained in the literature ${ }^{2}$.

\section{Conclusions}

The stability of dark breathers using of the symmetric Morse potential have been obtained with the Floquet's theory. It is very important to emphasize that dark breathers at low coupling are shown to be stable in the PBD model with $\mathrm{k}<0.004$. For $k=0.004$ we have harmonic bifurcation and the mobile dark breather. In this case and using numerical simulations we can demonstrate that the mean value of the hydrogen bond stretching is zero.

For the symmetric potentials we have significant fluctuations in the analysis of the breathing DNA with solvent and external potentials. The external potential is more important than the viscous force for the estimated melting temperature and the mean value of the hydrogen bond stretching.

\section{Acknowledgments}

The authors would like to acknowledge the Universidad Nacional del Callao-Facultad Ciencias de la Salud for the technological support. We would also like to thank Walter Julio Cortez Morales and Olga Estrada for economical support. 


\section{References}

[1] von Hippel, P., Johnson, N., Marcus, A., 50 years of DNA 'Breathing': Reflections on Old and New Approaches, Biopolymers 99 (12) (2013) 923-954. https://doi.org/10.1002/bip.22347.

[2] Hidayat, W., Sulaiman, A., Viridi, S, Zen, F. P., The viscous and external forces effect on the thermal denaturation of Peyrard-Bishop, Physical Chemistry \& Biophysics 5 (5) (2015) 1-5 (186). https://doi.org/10.4172/2161-0398.1000186.

[3] Flach, S., Gorbach, A., Discrete breathersAdvance in theory and applications, Physics Reports $467 \quad(1-3) \quad$ (2008) 1-116. https://doi.org/10.1016/j.physrep.2008.05.002.

[4] Mackay, R. S., Aubry, S., Proof of existence of breathers for time-reversible for Hamiltonian networks of weakly coupled oscillators, Nonlinearity 7 (1994) 1623-1643. https://doi.org/10.1088/0951-7715/7/6/006.

[5] Cuevas J., Localizacion y Transferencia de Energia en Redes Anarmónicas No Homogéneas, $\mathrm{Ph}$. D. Thesis, Universidad de Sevilla, Sevilla, España (2003). http://grupo.us.es/gfnl/thesis.htm.

[6] Aubry, S., Cretegny, T., Mobility and Reactivity of Discrete Breathers, Physica D 119 (12) (1998) 34-46. https://doi.org/10.1016/S01672789(98)00062-1.

[7] Ambjomsson, T., Banik, S. K., Krichevsky, O., Metzler, R., Breathing dynamics in Heteropolymer DNA, Biophysics J. 92 (8) (2007) 2674-2684. https://doi.org/10.1529/biophysj.106.095935.

[8] Cortez, H., Drigo Filho, E., Ruggiero, J. R., Breather stability in one dimensional Lattices with a symmetric Morse Potential, TEMA, Tend. Mat. Apl. Comput. 9 (2) (2008) 205-212. https://doi.org/10.5540/tema.2008.09.02.0205.

[9] Cortez, H., Drigo Filho, E., Ruggiero., J. R., Cortez, M., Mobile breathers in a nonlinear model for DNA breathing, Eclética Química Journal 42 (2017) 71-75. https://doi.org/10.26850/16784618eqj.42.1.2017.p71-75.

[10] Sulaiman, A., Zen, F. P., Alatas, H., Handoko, L. T., Dynamics of DNA breathing in the
Peyrard-Bishop weth damping and external force,

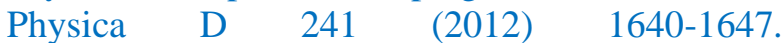
https://doi.org/10.1016/j.physd.2012.06.011.

[11] Cortez, H., Tese de doutorado, Modelo Dinâmico e estatístico aplicado à transição de fase. UNESP.(2009)

https://repositorio.unesp.br/bitstream/handle/1144 9/100461/cortezgutierrez_ho_dr_sjrp.pdf?sequenc $\mathrm{e}=1$ \&isAllowed $=\mathrm{y}$.

[12] Cortez, H., Drigo Filho, E., Ruggiero, J. R., Gutierrez, M. C., Fuentes Rivera, L. C., Thermodynamics of DNA with "hump" Morse potential, Eclética Química Journal 41 (2016) 60$65 . \quad$ https://doi.org/10.26850/1678 4618eqj.v41.1.2016.p60-65.

[13] Bustamante, C. Smith, S.B., Liphardt,J., Smith, D., Single-molecule studies of DNA mechanics, Current Opinion in Structural Biology $10 \quad$ (3) (2000) 279-285. https://doi.org/10.1016/S0959-440X(00)00085-3. 